\title{
Dirac Equation under Scalar, Vector, and Tensor Cornell Interactions
}

\author{
H. Hassanabadi, ${ }^{1}$ E. Maghsoodi, ${ }^{1}$ \\ S. Zarrinkamar, ${ }^{2}$ and H. Rahimov ${ }^{3}$ \\ ${ }^{1}$ Physics Department, Shahrood University of Technology, Shahrood 3619995161, Iran \\ 2 Young Researchers Club, Garmsar Branch, Islamic Azad University, Garmsar, Iran \\ ${ }^{3}$ Computer Engineering Department, Shahrood University of Technology, Shahrood, Iran
}

Correspondence should be addressed to E. Maghsoodi, e.maghsoodi184@gmail.com

Received 4 July 2012; Revised 27 August 2012; Accepted 29 August 2012

Academic Editor: S. H. Dong

Copyright $@ 2012 \mathrm{H}$. Hassanabadi et al. This is an open access article distributed under the Creative Commons Attribution License, which permits unrestricted use, distribution, and reproduction in any medium, provided the original work is properly cited.

Spin and pseudospin symmetries of Dirac equation are solved under scalar, vector, and tensor interactions for arbitrary quantum number via the analytical ansatz approach. The spectrum of the system is numerically reported for typical values of the potential parameters.

\section{Introduction}

No doubt, the Cornell potential (alternatively called Funnel in the literature) is, if not the best, among the most appealing interactions in particle physics. The Cornell potential contains a confining term besides the Coulomb interaction and has successfully accounted for the particle physics data [1]. Unfortunately, to our best knowledge, the potential does not possess exact solutions under all common equations of quantum mechanics, that is, the nonrelativistic Schrödinger equation, and relativistic Dirac, Klein-Gordon, Proca, and Duffin-KemmerPetiau (DKP) equations. Here, we focus on the relativistic symmetries of Dirac equation, that is, spin and pseudospin symmetries, which provide a reliable theoretical basis for hadronic and nuclear spectroscopy [2-12]. This has motivated many studies under various interactions within the past two decades ([13-18] and many references therein). Nevertheless, none of these papers has investigated the symmetry limits under the Cornell potential. This is definitely due to the complicated nature of the resulting differential equation which cannot be solved by common analytical techniques of quantum mechanics such as the supersymmetry quantum mechanics (SUSYQ), Lie groups, Nikiforov-Uvarov (NU) technique, and point canonical transformations, In our study, we make use of the ansatz approach to deal with this 
complicated equation. A survey on the application of this technique to other wave equations including Schrödinger, spinless-Salpeter, Dirac, Klein-Gordon, and DKP equations can be found in [19-27]. We organize the study as follows. In the first step, we review the most essential equations of the symmetry limits. We next propose a physical ansatz solution to the equation and, in a systematic manner, calculate the spectrum of the system for any arbitrary state. To provide a better understanding of the solutions, we provide some numerical data for the spectrum as well.

\section{Dirac Equation Including Tensor Coupling}

In spherical coordinates, Dirac equation with both scalar potential $S(r)$ and vector potential $V(r)$ is expressed as $[2,3]$

$$
[\vec{\alpha} \cdot \vec{p}+\beta(M+S(r))-i \beta \vec{\alpha} \cdot \hat{r} U(r)] \psi(\vec{r})=[E-V(r)] \psi(\vec{r}),
$$

where $E$ is the relativistic energy of the system; $\alpha$ and $\beta$ are the $4 \times 4$ Dirac matrices and $\vec{p}=-i \vec{\nabla}$ stands for the momentum operator. For a particle in a spherical field, the total angular momentum operator $\vec{J}$ and spin-orbit matrix operator $K=(\vec{\sigma} \cdot \vec{L}+1)$, where $\sigma$ and $L$ are, respectively, the Pauli matrix and orbital angular momentum, commute with the Dirac Hamiltonian. The eigenvalues of $K$ are $\kappa=-(j+1 / 2)$ for the aligned spin $\left(s_{1 / 2}, p_{3 / 2}\right.$, etc. $)$ and $\mathcal{K}=(j+1 / 2)$ for the unaligned spin $\left(p_{1 / 2}, d_{3 / 2}\right.$, etc.). The complete set of the conservative quantities can be chosen as $\left(H, K, J^{2}, J_{z}\right)$. As shown in [13], the Dirac spinor is considered as

$$
\psi_{n k}(\vec{r})=\left(\begin{array}{l}
f_{n k}(\vec{r}) \\
g_{n k}(\vec{r})
\end{array}\right)=\left(\begin{array}{l}
\frac{F_{n k}(\vec{r})}{r} Y_{j m}^{\ell}(\theta, \varphi) \\
i \frac{G_{n k}(\vec{r})}{r} Y_{j m}^{\tilde{\ell}}(\theta, \varphi)
\end{array}\right),
$$

where $F_{n k}(\vec{r})$ and $G_{n k}(\vec{r})$ are the radial wave functions of the upper and lower components, respectively, and $Y_{j m}^{\ell}(\theta, \varphi)$ and $Y_{j m}^{\tilde{\ell}}(\theta, \varphi)$, respectively, stand for spin and pseudospin spherical harmonics coupled to the angular momentum $j . m$ is the projection of the angular momentum on the $z$-axis. The orbital angular momentum quantum numbers $\ell$ and $\tilde{\ell}$ refer to the upper and lower components, respectively. The quasidegenerate doublet structure can be expressed in terms of pseudospin angular momentum $s=1 / 2$ and pseudoorbital angular momentum $\tilde{\ell}$, which is defined as $\tilde{\ell}=\ell+1$ for aligned spin $j=\tilde{\ell}-1 / 2$ and $\tilde{\ell}=\ell-1$ for unaligned spin $j=\tilde{\ell}+1 / 2$. As shown in [2,3], substitution of (2.2) into (2.1) yields the following two-coupled differential equations:

$$
\begin{aligned}
& \left(\frac{d}{d r}+\frac{\kappa}{r}-U(r)\right) F_{n k}(r)=\left(M+E_{n k}-V(r)+S(r)\right) G_{n k}(r), \\
& \left(\frac{d}{d r}-\frac{\kappa}{r}+U(r)\right) G_{n k}(r)=\left(M-E_{n k}+V(r)+S(r)\right) F_{n k}(r),
\end{aligned}
$$


which simply give

$$
\begin{aligned}
& \left\{\frac{d^{2}}{d r^{2}}-\frac{\kappa(\kappa+1)}{r^{2}}+\frac{2 \kappa}{r} U(r)-\frac{d U(r)}{d r}-U^{2}(r)+\frac{d \Delta(r) / d r}{M+E_{n \kappa}-\Delta(r)}\left(\frac{d}{d r}+\frac{\kappa}{r}-U(r)\right)\right\} F_{n \kappa}(r) \\
& =\left(M+E_{n \kappa}-\Delta(r)\right)\left(M-E_{n \kappa}+\Sigma(r)\right) F_{n \kappa}(r), \\
& \left\{\frac{d^{2}}{d r^{2}}-\frac{\kappa(\kappa-1)}{r^{2}}+\frac{2 \kappa}{r} U(r)+\frac{d U(r)}{d r}-U^{2}(r)-\frac{d \Sigma(r) / d r}{M-E_{n \kappa}+\Sigma(r)}\left(\frac{d}{d r}-\frac{\kappa}{r}+U(r)\right)\right\} G_{n \kappa}(r) \\
& =\left(M+E_{n \kappa}-\Delta(r)\right)\left(M-E_{n \kappa}+\Sigma(r)\right) G_{n \kappa}(r),
\end{aligned}
$$

where, as the notation indicates, $\Delta(r)=V(r)-S(r)$ and $\Sigma(r)=V(r)+S(r)$.

\subsection{Pseudospin Symmetry Limit}

Under the condition of the pseudospin symmetry, $d \Sigma(r) / d r=0$ or equivalently $\Sigma(r)=C_{g p s}=$ Const. $[2,3]$. We choose $\Delta(r)$ as the Cornell potential:

$$
\Delta(r)=a_{\mathrm{ps}} r+\frac{b_{\mathrm{ps}}}{r}
$$

For the tensor term, we consider the Cornell potential:

$$
U(r)=A r+\frac{B}{r}
$$

Substitution of these two terms in into (2.5) gives

$$
\begin{aligned}
& \left\{\frac{d^{2}}{d r^{2}}+\frac{1}{r^{2}}\left(-\kappa(\kappa-1)+2 \kappa B-B-B^{2}\right)-A^{2} r^{2}+\left(a_{\mathrm{ps}} M-a_{\mathrm{ps}} E_{n \kappa}^{p s}+a_{\mathrm{ps}} C_{\mathrm{ps}}\right) r\right. \\
& \left.+\frac{1}{r}\left(b_{\mathrm{ps}} M-b_{\mathrm{ps}} E_{n \kappa}^{\mathrm{ps}}+b_{\mathrm{ps}} C_{\mathrm{ps}}\right)-M^{2}-M C_{\mathrm{ps}}+\left(E_{n \kappa}^{\mathrm{ps}}\right)^{2}-E_{n \kappa}^{\mathrm{ps}} C_{\mathrm{ps}}+2 \kappa A+A-2 A B\right\} \\
& \quad \times G_{n \kappa}^{p s}(r)=0,
\end{aligned}
$$

where $\kappa=-\tilde{\ell}$ and $\kappa=\tilde{\ell}+1$ for $\kappa<0$ and $\kappa>0$, respectively. 


\subsection{Spin Symmetry Limit}

In the spin symmetry limit $d \Delta(r) / d r=0$ or $\Delta(r)=C_{g s}=$ const. [2,3]. As the previous section, we consider

$$
\begin{aligned}
& \Sigma(r)=a_{s} r+\frac{b_{s}}{r}, \\
& U(r)=A r+\frac{B}{r} .
\end{aligned}
$$

Substitution of the latter in (2.4) gives

$$
\begin{aligned}
& \left\{\frac{d^{2}}{d r^{2}}+\frac{1}{r^{2}}\left(-\kappa(\kappa+1)+2 \kappa B+B-B^{2}\right)-A^{2} r^{2}+\left(-a_{s} M-a_{s} E_{n \kappa}^{s}+a_{s} C_{s}\right) r\right. \\
& \left.\quad+\frac{1}{r}\left(-b_{s} M-b_{s} E_{n \kappa}^{S}+b_{s} C_{s}\right)-M^{2}+M C_{s}+\left(E_{n \kappa}^{\mathrm{ps}}\right)^{2}-E_{n \kappa}^{S} C_{s}+2 \kappa A-A-2 A B\right\} F_{n \kappa}^{S}(r)=0,
\end{aligned}
$$

where $\kappa=\ell$ and $\kappa=-\ell-1$ for $\kappa<0$ and $\kappa>0$, respectively.

\section{The Ansatz Solution}

\subsection{Solution of the Pseudospin Symmetry Limit}

In the previous section, we obtained a Schrödinger-like equation of the form

$$
\frac{d^{2} G_{n \kappa}^{\mathrm{ps}}(r)}{d r^{2}}+\left[\varepsilon_{n \kappa}^{\mathrm{ps}}-a_{1}^{\mathrm{ps}} r^{2}-b_{1}^{p s} r+\frac{c_{1}^{\mathrm{ps}}}{r}+\frac{d_{1}^{\mathrm{ps}}-\kappa(\kappa-1)}{r^{2}}\right] G_{n \kappa}^{\mathrm{ps}}(r)=0
$$

where

$$
\begin{gathered}
\varepsilon_{n \kappa}^{\mathrm{ps}}=-M^{2}-M C_{\mathrm{ps}}+\left(E_{n \kappa}^{\mathrm{ps}}\right)^{2}-E_{n \kappa}^{\mathrm{ps}} C_{\mathrm{ps}}+2 \kappa A+A-2 A B, \\
a_{1}^{\mathrm{ps}}=A^{2}, \\
b_{1}^{\mathrm{ps}}=-a_{\mathrm{ps}} M+a_{\mathrm{ps}} E_{n \kappa}^{\mathrm{ps}}-a_{\mathrm{ps}} C_{\mathrm{ps}} \\
c_{1}^{\mathrm{ps}}=b_{\mathrm{ps}} M-b_{\mathrm{ps}} E_{n \kappa}^{\mathrm{ps}}+b_{\mathrm{ps}} C_{\mathrm{ps}} \\
d_{1}^{\mathrm{ps}}=2 \kappa B-B-B^{2} .
\end{gathered}
$$


Equation (3.1) fails to admit exact analytical solutions. Therefore, we follow the ansatz approach with the starting square:

$$
G_{n \kappa}^{\mathrm{ps}}(r)=f_{n}^{\mathrm{ps}}(r) \exp \left(g_{\kappa}^{\mathrm{ps}}(r)\right)
$$

where

$$
\begin{gathered}
f_{n}^{\mathrm{ps}}(r)= \begin{cases}1, & \text { if } n=0, \\
\prod_{i=1}^{n}\left(r-\alpha_{i}^{n}\right), & \text { if } n \geq 1,\end{cases} \\
g_{\kappa}^{\mathrm{ps}}(r)=-\frac{1}{2} \alpha^{\mathrm{ps}} r^{2}-\beta^{\mathrm{ps}} r+\delta^{\mathrm{ps}} \ln \mathrm{r}, \quad \alpha^{\mathrm{ps}}>0, \beta^{\mathrm{ps}}>0 .
\end{gathered}
$$

By substitution of $f_{n}(r)$ and $g_{\kappa}(r)$ into (3.3), we find

$$
G_{n \kappa}^{\mathrm{ps}^{\prime \prime}}(r)=\left[g_{\kappa}^{\mathrm{ps} \prime}(r)+g_{\kappa}^{\mathrm{ps}^{\prime 2}}(r)+\frac{f_{n}^{\mathrm{ps}^{\prime \prime}}(r)+2 g_{\kappa}^{\mathrm{ps}^{\prime}}(r) f_{n}^{\mathrm{ps}^{\prime}}(r)}{f_{n}^{\mathrm{ps}}(r)}\right] G_{n \kappa}^{\mathrm{ps}}(r) .
$$

Here, we consider the case $n=0$. From (3.4)-(3.6) we find

$$
\begin{aligned}
& G_{0 \kappa}^{\mathrm{ps} \prime}(r) \\
& \quad=\left\{\left(\alpha^{\mathrm{ps}}\right)^{2} r^{2}+2 \alpha^{\mathrm{ps}} \beta^{\mathrm{ps}} r-\alpha^{\mathrm{ps}}\left[1+2\left(\delta^{\mathrm{ps}}+0\right)\right]+\left(\beta^{\mathrm{ps}}\right)^{2}-\frac{2 \beta^{\mathrm{ps}}\left(\delta^{\mathrm{ps}}+0\right)}{r}+\frac{\delta^{\mathrm{ps}}\left(\delta^{\mathrm{ps}}-1\right)}{r^{2}}\right\} G_{0 \kappa}^{\mathrm{ps}}(r) .
\end{aligned}
$$

By comparing the corresponding powers of (3.1) and (3.7), we have

$$
\begin{gathered}
\alpha^{\mathrm{ps}}=\sqrt{a_{1}^{\mathrm{ps}}}, \quad a_{1}^{\mathrm{ps}}>0, \\
\beta^{\mathrm{ps}}=\frac{b_{1}^{\mathrm{ps}}}{2 \sqrt{a_{1}^{\mathrm{ps}}}}, \\
c_{1}^{\mathrm{ps}}=2 \beta^{\mathrm{ps}}\left(\delta^{\mathrm{ps}}+0\right), \\
\delta^{\mathrm{ps}}=\frac{1}{2}\left(1 \pm \sqrt{(2 \kappa-1)^{2}-4 d_{1}^{\mathrm{ps}}}\right)=\frac{1}{2}\left(1 \pm k_{1}^{\mathrm{ps}}\right), \\
\varepsilon_{0 \kappa}^{\mathrm{ps}}=\alpha^{\mathrm{ps}}\left[1+2\left(\delta^{\mathrm{ps}}+0\right)\right]-\left(\beta^{\mathrm{ps}}\right)^{2},
\end{gathered}
$$

where $k_{1}^{\mathrm{ps}}=\sqrt{(2 \kappa-1)^{2}-4 d_{1}^{\mathrm{ps}}}$. Actually, to have well-behaved solutions of the radial wave function at boundaries, namely, the origin and the infinity, we need to take $\delta$ from (3.8) as

$$
\delta^{\mathrm{ps}}=\frac{1}{2}\left(1+k_{1}^{\mathrm{ps}}\right) .
$$


Form (3.2), (3.8), the ground-state energy satisfies

$$
-M^{2}-M C_{\mathrm{ps}}+\left(E_{0 \kappa}^{\mathrm{ps}}\right)^{2}-E_{0 \kappa}^{\mathrm{ps}} C_{p s}+2 \kappa A+A-2 A B-\alpha^{\mathrm{ps}}\left[1+2\left(\delta^{\mathrm{ps}}+0\right)\right]+\beta^{\mathrm{ps} 2}=0
$$

or

$$
\left(E_{0 \kappa}^{\mathrm{ps}}\right)^{2}-E_{0 \kappa}^{\mathrm{ps}} C_{\mathrm{ps}}-M^{2}-M C_{\mathrm{ps}}+2 \kappa A+A-2 A B-\sqrt{a_{1}^{\mathrm{ps}}}\left(2+\sqrt{(2 \kappa-1)^{2}-4 d_{1}^{\mathrm{ps}}}\right)+\frac{b_{1}^{\mathrm{ps} 2}}{4 a_{1}^{\mathrm{ps}}}=0
$$

which is more compactly written as

$$
\begin{gathered}
E_{0 \kappa}^{\mathrm{ps} \pm}=\frac{1}{2}\left\{C_{\mathrm{ps}} \pm\left[C_{\mathrm{ps}}^{2}-8 \kappa A-4 A+8 A B+4 M^{2}+4 M C_{\mathrm{ps}}+8 \sqrt{a_{1}^{\mathrm{ps}}}\right.\right. \\
\left.\left.+4 \sqrt{a_{1}^{\mathrm{ps}}} \sqrt{4 \kappa^{2}-4 \kappa+1-4 d_{1}^{\mathrm{ps}}}-4 \beta^{\mathrm{ps} 2}\right]^{1 / 2}\right\},
\end{gathered}
$$

where the parameter $a_{\mathrm{ps}}$ of potential (2.6) from (3.8) should satisfy the following restriction:

$$
a_{\mathrm{ps}}=\frac{-2 b_{\mathrm{ps}} \sqrt{a_{1}^{\mathrm{ps}}}}{\left(1+\sqrt{(2 \kappa-1)^{2}-4 d_{1}^{\mathrm{ps}}}\right)}=\frac{-2 b_{\mathrm{ps}} \sqrt{a_{1}^{\mathrm{ps}}}}{\left(1+k_{1}^{\mathrm{ps}}\right)}
$$

From (3.3), (3.4), and (3.8), the upper and lower components of the wave function are

$$
\begin{aligned}
& g_{\kappa}(r)=-\frac{1}{2} \sqrt{a_{1}^{\mathrm{ps}}} r^{2}-\frac{b_{1}^{\mathrm{ps}}}{2 \sqrt{a_{1}^{\mathrm{ps}}}} r+\frac{1}{2}\left(1+\sqrt{(2 \kappa-1)^{2}-4 d_{1}^{\mathrm{ps}}}\right) \ln r \\
& G_{0 \kappa}^{\mathrm{ps}}(r)=N_{0 \kappa} r^{(1 / 2)\left(1+\sqrt{\left.(2 \kappa-1)^{2}-4 d_{1}^{\mathrm{ps}}\right)}\right.} \exp \left(-\frac{1}{2} \sqrt{a_{1}^{\mathrm{ps}}} r^{2}-\frac{b_{1}^{\mathrm{ps}}}{2 \sqrt{a_{1}^{\mathrm{ps}}}} r\right) \\
& F_{0 \kappa}^{\mathrm{ps}}(r)=\frac{1}{M-E_{0 \kappa}^{\mathrm{ps}}+C_{\mathrm{ps}}}\left(\frac{d}{d r}-\frac{\kappa}{r}+U(r)\right) G_{0 \kappa}^{\mathrm{ps}}(r) .
\end{aligned}
$$


Advances in High Energy Physics

For the first node $(n=1)$, using $f_{1}(r)=\left(r-\alpha_{1}^{(1)}\right)$ and $g_{\kappa}(r)$ from (3.5), we arrive at

$$
\begin{aligned}
a_{1}^{\mathrm{ps}} r^{2}+ & b_{1}^{\mathrm{ps}} r-\frac{c_{1}^{\mathrm{ps}}}{r}+\frac{\kappa(\kappa-1)-d_{1}^{\mathrm{ps}}}{r^{2}}-\varepsilon_{1 \kappa}^{\mathrm{ps}} \\
= & -\alpha^{\mathrm{ps}}-\frac{\delta^{\mathrm{ps}}}{r^{2}}+\left(\alpha^{\mathrm{ps}}\right)^{2} r^{2}+\left(\beta^{\mathrm{ps}}\right)^{2}+\frac{\left(\delta^{\mathrm{ps}}\right)^{2}}{r^{2}}+2 \alpha^{\mathrm{ps}} \beta^{\mathrm{ps}} r-2 \delta^{\mathrm{ps}} \alpha^{\mathrm{ps}}-\frac{2 \beta^{\mathrm{ps}} \delta^{\mathrm{ps}}}{r} \\
& +\frac{-2 \alpha^{\mathrm{ps}} r-2 \beta^{\mathrm{ps}}+2 \delta^{\mathrm{ps}} / r}{r-\alpha_{1}^{(1)}} .
\end{aligned}
$$

Here, the consequent relations between the potential parameters and the coefficients $\alpha^{\mathrm{ps}}, \beta^{\mathrm{ps}}$, $\delta^{\mathrm{ps}}$, and $\alpha_{1}^{(1)}$ are

$$
\begin{aligned}
& \alpha^{\mathrm{ps}}=\sqrt{a_{1}^{\mathrm{ps}}}, \quad \beta^{\mathrm{ps}}=\frac{b_{1}^{\mathrm{ps}}}{2 \sqrt{a_{1}^{\mathrm{ps}}}}, \quad \delta^{\mathrm{ps}}=\frac{1}{2}\left(1+k_{1}^{\mathrm{ps}}\right), \\
& \varepsilon_{1 \kappa}^{\mathrm{ps}}=\alpha^{\mathrm{ps}}\left[1+2\left(\delta^{\mathrm{ps}}+1\right)\right]-\left(\beta^{\mathrm{ps}}\right)^{2}, \\
& \left(c_{1}^{\mathrm{ps}}-2 \beta^{\mathrm{ps}} \delta^{\mathrm{ps}}\right) \alpha_{1}^{(1)}=2 \delta^{\mathrm{ps}}, \\
& c_{1}^{\mathrm{ps}}-2 \beta^{\mathrm{ps}}\left(\delta^{\mathrm{ps}}+1\right)=2 \alpha^{\mathrm{ps}} \alpha_{1}^{(1)} \text {. }
\end{aligned}
$$

By solving the above equations one can find $c_{1}^{\mathrm{ps}}$ and $\alpha_{1}^{(1)}$ as

$$
\begin{gathered}
c_{1}^{\mathrm{ps}}=\frac{b_{1}^{\mathrm{ps}}}{2 \sqrt{a_{1}^{\mathrm{ps}}}}\left(\sqrt{(2 \kappa-1)^{2}-4 d_{1}^{\mathrm{ps}}}+2\right)+\sqrt{\frac{b_{1}^{\mathrm{ps} 2}}{4 a_{1}^{\mathrm{ps}}}+2 \sqrt{a_{1}^{\mathrm{ps}}}\left(1+\sqrt{(2 \kappa-1)^{2}-4 d_{1}^{\mathrm{ps}}}\right)} \\
\alpha^{\mathrm{ps}} \alpha_{1}^{(1) 2}+\beta^{\mathrm{ps}} \alpha_{1}^{(1)}-\delta^{\mathrm{ps}}=0, \\
\longrightarrow \alpha_{1}^{(1)}=\frac{1}{2} \frac{-\beta^{\mathrm{ps}}+\sqrt{\left(\beta^{\mathrm{ps}}\right)^{2}+4 \alpha^{\mathrm{ps}} \delta^{\mathrm{ps}}}}{\alpha^{\mathrm{ps}}}=\frac{-b_{1}^{\mathrm{ps}}}{4 a_{1}^{\mathrm{ps}}}+\sqrt{\frac{b_{1}^{\mathrm{ps} 2}}{16 a_{1}^{\mathrm{ps}}}+\frac{1+\sqrt{(2 \kappa-1)^{2}-4 d_{1}^{\mathrm{ps}}}}{2 \sqrt{a_{1}^{\mathrm{ps}}}}} .
\end{gathered}
$$

The energy eigenvalues therefore are

$$
\begin{gathered}
E_{1 \kappa}^{\mathrm{ps} \pm}=\frac{1}{2}\left\{C_{\mathrm{ps}} \pm\left[C_{\mathrm{ps}}^{2}-8 \kappa A-4 A+8 A B+4 M^{2}+4 M C_{\mathrm{ps}}+16 \sqrt{a_{1}^{\mathrm{ps}}}\right.\right. \\
\left.\left.+4 \sqrt{a_{1}^{\mathrm{ps}}} \sqrt{4 \kappa^{2}-4 \kappa+1-4 d_{1}^{\mathrm{ps}}}-4 \beta^{\mathrm{ps} 2}\right]^{1 / 2}\right\}
\end{gathered}
$$


where the parameter $a_{\mathrm{ps}}$ from (3.8) is

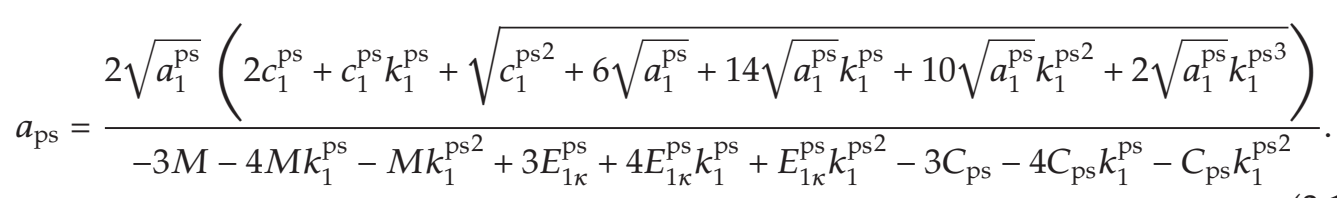

For the upper and lower components of the wave function we thus have

$$
\begin{gathered}
G_{1 \kappa}^{\mathrm{ps}}(r)=N_{1 \kappa}\left(r-\alpha_{1}^{(1)}\right) r^{(1 / 2)\left(1+\sqrt{(2 \kappa-1)^{2}-4 d_{1}^{\mathrm{ps}}}\right)} \exp \left(-\frac{1}{2} \sqrt{a_{1}^{\mathrm{ps}}} r^{2}-\frac{b_{1}^{\mathrm{ps}}}{2 \sqrt{a_{1}^{\mathrm{ps}}}} r\right) \\
F_{1 \kappa}^{\mathrm{ps}}(r)=\frac{1}{M-E_{1 \kappa}^{\mathrm{ps}}+C_{\mathrm{ps}}}\left(\frac{d}{d r}-\frac{\kappa}{r}+U(r)\right) G_{1 \kappa}^{\mathrm{ps}}(r) .
\end{gathered}
$$

Following the analytic iteration procedures for the second node $(n=2)$ with $f_{2}(r)=$ $\left(r-\alpha_{1}^{(2)}\right)\left(r-\alpha_{2}^{(2)}\right)$ and $g_{\kappa}(r)$ as defined in (3.6), the relations between the potential parameters and the coefficients $\alpha^{\mathrm{ps}}, \beta^{\mathrm{ps}}, \delta^{\mathrm{ps}}, \alpha_{1}^{(2)}$, and $\alpha_{2}^{(2)}$ are

$$
\begin{gathered}
\alpha^{\mathrm{ps}}=\sqrt{a_{1}^{\mathrm{ps}}}, \quad \beta^{\mathrm{ps}}=\frac{b_{1}^{\mathrm{ps}}}{2 \sqrt{a_{1}^{\mathrm{ps}}}}, \quad \delta^{\mathrm{ps}}=\frac{1}{2}\left(1+k_{1}^{\mathrm{ps}}\right), \\
\varepsilon_{2 \kappa}^{\mathrm{ps}}=\alpha^{\mathrm{ps}}\left[1+2\left(\delta^{\mathrm{ps}}+2\right)\right]-\left(\beta^{\mathrm{ps}}\right)^{2}, \\
c_{1}^{\mathrm{ps}}-2 \beta^{\mathrm{ps}}\left(\delta^{\mathrm{ps}}+2\right)=2 \alpha^{\mathrm{ps}} \sum_{i=1}^{2} \alpha_{i}^{(2)}, \quad\left(c_{1}^{\mathrm{ps}}-2 \beta^{\mathrm{ps}} \delta^{\mathrm{ps}}\right) \sum_{i<j}^{2} \alpha_{i}^{(2)} \alpha_{j}^{(2)}=2 \delta^{\mathrm{ps}} \sum_{i=1}^{2} \alpha_{i}^{(2)}, \\
{\left[c_{1}^{\mathrm{ps}}-2 \beta^{\mathrm{ps}}\left(\delta^{\mathrm{ps}}+1\right)\right] \sum_{i=1}^{2} \alpha_{i}^{(2)}=4 \alpha^{\mathrm{ps}} \sum_{i<j}^{2} \alpha_{i}^{(2)} \alpha_{j}^{(2)}+2\left(2 \delta^{\mathrm{ps}}+1\right) .}
\end{gathered}
$$

The coefficients $\alpha_{1}^{(2)}$ and $\alpha_{2}^{(2)}$ are found from the constraint relations [20, 27-29]:

$$
\begin{gathered}
\alpha^{\mathrm{ps}} \sum_{i=1}^{2} \alpha_{i}^{(2) 2}+\beta^{\mathrm{ps}} \sum_{i=1}^{2} \alpha_{i}^{(2)}-\left(2 \delta^{\mathrm{ps}}+1\right)=0 \\
\delta^{\mathrm{ps}} \sum_{i=1}^{2} \alpha_{i}^{(2) 2}-\left(\beta^{\mathrm{ps}} \sum_{i=1}^{2} \alpha_{i}^{(2)}+1\right) \sum_{j<k}^{2} \alpha_{j}^{(2)} \alpha_{k}^{(2)}-2 \alpha^{\mathrm{ps}} \sum_{j<k}^{2} \alpha_{j}^{(2) 2} \alpha_{k}^{(2) 2}=0 .
\end{gathered}
$$


Therefore, the energy eigenvalue in this case is

$$
\begin{gathered}
E_{2 \kappa}^{\mathrm{ps} \pm}=\frac{1}{2}\left\{C_{\mathrm{ps}} \pm[\right. \\
C_{\mathrm{ps}}^{2}-8 \kappa A-4 A+8 A B+4 M^{2}+4 M C_{\mathrm{ps}}+24 \sqrt{a_{1}^{\mathrm{ps}}} \\
\left.\left.+4 \sqrt{a_{1}^{\mathrm{ps}}} \sqrt{4 \kappa^{2}-4 \kappa+1-4 d_{1}^{\mathrm{ps}}}-4 \beta^{\mathrm{ps} 2}\right]^{1 / 2}\right\}
\end{gathered}
$$

and the lower component of the wave function is

$$
G_{2 \kappa}^{\mathrm{ps}}(r)=N_{2 \kappa} \prod_{i=1}^{2}\left(r-\alpha_{i}^{(2)}\right) r^{(1 / 2)\left(1+\sqrt{\left.(2 \kappa-1)^{2}-4 d_{1}^{\mathrm{ps}}\right)}\right.} \exp \left(-\frac{1}{2} \sqrt{a_{1}^{\mathrm{ps}}} r^{2}-\frac{b_{1}^{\mathrm{ps}}}{2 \sqrt{a_{1}^{\mathrm{ps}}}} r\right)
$$

\subsection{Solution of the Spin Symmetry Limit}

In this case, our ordinary differential equation is

$$
\frac{d^{2} F_{n \kappa}^{s}(r)}{d r^{2}}+\left[\varepsilon_{n \kappa}^{s}-a_{1}^{s} r^{2}-b_{1}^{s} r+\frac{c_{1}^{s}}{r}+\frac{d_{1}^{s}-\kappa(\kappa+1)}{r^{2}}\right] F_{n \kappa}^{s}(r)=0,
$$

with

$$
\begin{gathered}
\varepsilon_{n \kappa}^{s}=-M^{2}+M C_{s}+\left(E_{n \kappa}^{s}\right)^{2}-E_{n \kappa}^{s} C_{s}+2 \kappa A-A-2 A B, \\
a_{1}^{s}=A^{2} \\
b_{1}^{s}=a_{s} M+a_{s} E_{n \kappa}^{s}-a_{s} C_{s} \\
c_{1}^{s}=-b_{s} M-b_{s} E_{n \kappa}^{s}+b_{s} C_{s} \\
d_{1}^{s}=2 \kappa B+B-B^{2}
\end{gathered}
$$

which cannot be solved by our common exact analytical techniques. Let us propose the ansatz solution:

$$
F_{n \kappa}^{S}(r)=f_{n}^{S}(r) \exp \left(g_{\kappa}^{S}(r)\right),
$$

where

$$
\begin{gathered}
f_{n}^{s}(r)= \begin{cases}1, & \text { if } n=0, \\
\prod_{i=1}^{n}\left(r-\alpha_{i}^{n}\right), & \text { if } n \geq 1,\end{cases} \\
g_{\kappa}^{s}(r)=-\frac{1}{2} \alpha^{s} r^{2}-\beta^{s} r+\delta^{s} \ln r, \quad \alpha^{s}>0, \beta^{s}>0 .
\end{gathered}
$$


By substitution of $f_{n}(r)$ and $g_{\kappa}(r)$ into (3.27), we find

$$
F_{n \kappa}^{s}{ }^{\prime \prime}(r)=\left[g_{\mathcal{\kappa}}^{s^{\prime \prime}}(r)+g_{\kappa}^{s^{\prime 2}}(r)+\frac{f_{n}^{s^{\prime \prime}}(r)+2 g_{\kappa}^{s^{\prime}}(r) f_{n}^{s^{\prime}}(r)}{f_{n}^{s}(r)}\right] F_{n \mathcal{K}}^{s}(r) .
$$

For the case of $n=0$, from (3.26)-(3.29), we find

$$
F_{n \kappa}^{s \prime \prime}(r)=\left\{\left(\alpha^{s}\right)^{2} r^{2}+2 \alpha^{s} \beta^{s} r-\alpha^{s}\left[1+2\left(\delta^{s}+0\right)\right]+\left(\beta^{s}\right)^{2}-\frac{2 \beta^{s}\left(\delta^{s}+0\right)}{r}+\frac{\delta^{s}\left(\delta^{s}-1\right)}{r^{2}}\right\} F_{n \kappa}^{s}(r) .
$$

By comparing the corresponding powers of (3.27) and (3.33), we have

$$
\begin{gathered}
\alpha^{s}=\sqrt{a_{1}^{s}}, \\
\beta^{s}=\frac{b_{1}^{s}}{2 \sqrt{a_{1}^{s}}}, \quad a_{1}^{s}>0, \\
c_{1}^{s}=2 \beta^{s}\left(\delta^{s}+0\right), \\
\delta^{s}=\frac{1}{2}\left(1 \pm \sqrt{(2 \kappa+1)^{2}-4 d_{1}^{s}}\right)=\frac{1}{2}\left(1 \pm k_{1}^{s}\right), \\
\varepsilon_{0 \kappa}^{s}=\alpha\left[1+2\left(\delta^{s}+0\right)\right]-\left(\beta^{s}\right)^{2},
\end{gathered}
$$

where $k_{1}^{s}=\sqrt{(2 \kappa+1)^{2}-4 d_{1}^{s}}$. To have physically acceptable solutions, we pick up the value

$$
\delta^{s}=\frac{1}{2}\left(1+k_{1}^{s}\right)
$$

By considering (3.26), (3.32), the first node eigenvalue satisfies

$$
-M^{2}+M C_{s}+\left(E_{n \kappa}^{s}\right)^{2}-E_{n \kappa}^{s} C_{s}+2 \kappa A-A-2 A B-\alpha^{s}\left[1+2\left(\delta^{s}+0\right)\right]+\left(\beta^{s}\right)^{2}=0,
$$

or equivalently

$$
\begin{gathered}
E_{0 \kappa}^{s \pm}=\frac{1}{2}\left\{C_{s} \pm\left[C_{s}^{2}+4 M^{2}-4 M C_{s}-8 \kappa A+4 A+8 A B+8 \sqrt{a_{1}^{s}}\right.\right. \\
\left.\left.+4 \sqrt{a_{1}^{s}} \sqrt{4 \kappa^{2}+4 \kappa+1-4 d_{1}^{s}}-4 \beta^{s 2}\right]^{1 / 2}\right\}
\end{gathered}
$$


where the parameter $a_{s}$ of potential (2.9a) should satisfy the restriction

$$
a_{s}=\frac{-2 b_{s} \sqrt{a_{1}^{s}}}{\left(1+k_{1}^{s}\right)} \text {. }
$$

From (3.27), (3.28), and (3.32), the upper and lower components of the wave function are

$$
\begin{gathered}
g_{\kappa}(r)=-\frac{1}{2} \sqrt{a_{1}^{s}} r^{2}-\frac{b_{1}^{s}}{2 \sqrt{a_{1}^{s}}} r+\frac{1}{2}\left(1+\sqrt{(2 \kappa+1)^{2}-4 d_{1}^{s}}\right) \ln r \\
F_{0 \kappa}^{s}(r)=N_{0 \kappa} r^{(1 / 2)\left(1+\sqrt{\left.(2 \kappa+1)^{2}-4 d_{1}^{s}\right)}\right.} \exp \left(-\frac{1}{2} \sqrt{a_{1}^{s}} r^{2}-\frac{b_{1}^{s}}{2 \sqrt{a_{1}^{s}}} r\right), \\
G_{0 \kappa}^{s}(r)=\frac{1}{M+E_{0 \kappa}^{s}-C_{s}}\left(\frac{d}{d r}+\frac{\kappa}{r}-U(r)\right) F_{0 \kappa}^{s}(r) .
\end{gathered}
$$

Secondly, for the first node $(n=1)$, using $f_{1}(r)=\left(r-\alpha_{1}^{(1)}\right)$ and $g_{\kappa}(r)$ from (3.28)-(3.30), our resulting equation is

$$
\begin{aligned}
a_{1}^{s} r^{2} & +b_{1}^{s} r-\frac{c_{1}^{s}}{r}+\frac{-d_{1}^{s}+\kappa(\kappa+1)}{r^{2}}-\varepsilon_{n \kappa}^{s} \\
& =-\alpha^{s}-\frac{\delta^{s}}{r^{2}}+\left(\alpha^{s}\right)^{2} r^{2}+\left(\beta^{s}\right)^{2}+\frac{\left(\delta^{s}\right)^{2}}{r^{2}}+2 \alpha^{s} \beta^{s} r-2 \delta^{s} \alpha^{s}-\frac{2 \beta^{s} \delta^{s}}{r}+\frac{-2 \alpha^{s} r-2 \beta^{s}+2 \delta^{s} / r}{r-\alpha_{1}^{(1)}} .
\end{aligned}
$$

The relations between the potential parameters and the coefficients $\alpha^{\mathrm{s}}, \beta^{\mathrm{s}}, \delta^{\mathrm{s}}$, and $\alpha_{1}^{(1)}$ are

$$
\begin{gathered}
\alpha^{s}=\sqrt{a_{1}^{s}}, \quad \beta^{s}=\frac{b_{1}^{s}}{2 \sqrt{a_{1}^{\mathrm{ps}}}}, \quad a_{1}^{s}>0, \quad \delta^{s}=\frac{1}{2}\left(1+\sqrt{(2 \kappa+1)^{2}-4 d_{1}^{s}}\right)=\frac{1}{2}\left(1+k_{1}^{s}\right), \\
\varepsilon_{1 \kappa}^{s}=\alpha^{s}\left[1+2\left(\delta^{s}+1\right)\right]-\left(\beta^{s}\right)^{2}, \\
\left(c_{1}^{s}-2 \beta^{s} \delta^{s}\right) \alpha_{1}^{(1)}=2 \delta^{s}, \\
c_{1}^{s}-2 \beta^{s}\left(\delta^{s}+1\right)=2 \alpha^{s} \alpha_{1}^{(1)},
\end{gathered}
$$


where $c_{1}^{s}$ and $\alpha_{1}^{(1)}$ are found from (3.41) as [20, 27-29]

$$
\begin{gathered}
c_{1}^{s}=\frac{b_{1}^{s}}{2 \sqrt{a_{1}^{s}}}\left(\sqrt{(2 \kappa+1)^{2}-4 d_{1}^{s}}+2\right)+\sqrt{\frac{b_{1}^{s 2}}{4 a_{1}^{s}}+2 \sqrt{a_{1}^{s}}\left(1+\sqrt{(2 \kappa+1)^{2}-4 d_{1}^{s}}\right)}, \\
\alpha^{s} \alpha_{1}^{(1) 2}+\beta^{s} \alpha_{1}^{(1)}-\delta^{s}=0
\end{gathered}
$$

or

$$
\alpha_{1}^{(1)}=\frac{1}{2} \frac{-\beta^{s}+\sqrt{\left(\beta^{s}\right)^{2}+4 \alpha^{s} \delta^{s}}}{\alpha^{s}}=\frac{-b_{1}^{s}}{4 a_{1}^{s}}+\sqrt{\frac{b_{1}^{s 2}}{16 a_{1}^{s 2}}+\frac{1+\sqrt{(2 \kappa+1)^{2}-4 d_{1}^{s}}}{2 \sqrt{a_{1}^{s}}}},
$$

which determine the corresponding energy as

$$
\begin{gathered}
E_{1 \kappa}^{s \pm}=\frac{1}{2}\left\{C_{s} \pm\left[C_{s}^{2}+4 M^{2}-4 M C_{s}-8 \kappa A+4 A+8 A B+16 \sqrt{a_{1}^{s}}\right.\right. \\
\left.\left.+4 \sqrt{a_{1}^{s}} \sqrt{4 \kappa^{2}+4 \kappa+1-4 d_{1}^{s}}-4 \beta^{s 2}\right]^{1 / 2}\right\}
\end{gathered}
$$

with

$$
a_{s}=\frac{2 \sqrt{a_{1}^{s}}\left(2 c_{1}^{s}+c_{1}^{s} k_{1}^{s}+\sqrt{c_{1}^{s 2}+6 \sqrt{a_{1}^{s}}+14 \sqrt{a_{1}^{s}} k_{1}^{s}+10 \sqrt{a_{1}^{s}} k_{1}^{s 2}+2 \sqrt{a_{1}^{s}} k_{1}^{s 3}}\right)}{3 M+4 M k_{1}^{s}+M k_{1}^{s 2}+3 E_{1 \kappa}^{s}+4 E_{1 \kappa}^{s} k_{1}^{s}+E_{1 \kappa}^{s} k_{1}^{s 2}-3 C_{s}-4 C_{s} k_{1}^{s}-C_{s} k_{1}^{s 2}} .
$$

The upper and lower components of the wave function are then simply found to be

$$
\begin{gathered}
F_{1 \kappa}^{s}(r)=N_{1 \kappa}\left(r-\alpha_{1}^{(1)}\right) r^{(1 / 2)\left(1+\sqrt{\left.(2 \kappa+1)^{2}-4 d_{1}^{s}\right)}\right.} \exp \left(-\frac{1}{2} \sqrt{a_{1}^{s}} r^{2}-\frac{b_{1}^{s}}{2 \sqrt{a_{1}^{s}}} r\right), \\
G_{1 \kappa}^{s}(r)=\frac{1}{M+E_{1 \kappa}^{s}-C_{s}}\left(\frac{d}{d r}+\frac{\kappa}{r}-U(r)\right) F_{1 \kappa}^{s}(r) .
\end{gathered}
$$


For the second node $(n=2)$, we choose $f_{2}(r)=\left(r-\alpha_{1}^{(2)}\right)\left(r-\alpha_{2}^{(2)}\right)$ and $g_{\kappa}(r)$ as defined in (3.28) and (3.29). The relations between the potential parameters and the coefficients $\alpha^{s}, \beta^{s}, \delta^{s}, \alpha_{1}^{(2)}$, and $\alpha_{2}^{(2)}$ are

$$
\begin{gathered}
\alpha^{s}=\sqrt{a_{1}^{s}}, \quad \beta^{s}=\frac{b_{1}^{s}}{2 \sqrt{a_{1}^{s}}}, \quad \delta^{s}=\frac{1}{2}\left(1+k_{1}^{s}\right), \\
\varepsilon_{2 \kappa}^{s}=\alpha^{s}\left[1+2\left(\delta^{s}+2\right)\right]-\left(\beta^{s}\right)^{2}, \\
c_{1}^{s}-2 \beta^{s}\left(\delta^{s}+2\right)=2 \alpha^{s} \sum_{i=1}^{2} \alpha_{i}^{(2)}, \quad\left(c_{1}^{s}-2 \beta^{s} \delta^{s}\right) \sum_{i<j}^{2} \alpha_{i}^{(2)} \alpha_{j}^{(2)}=2 \delta^{s} \sum_{i=1}^{2} \alpha_{i}^{(2)}, \\
{\left[c_{1}^{s}-2 \beta^{s}\left(\delta^{s}+1\right)\right] \sum_{i=1}^{2} \alpha_{i}^{(2)}=4 \alpha \sum_{i<j}^{2} \alpha_{i}^{(2)} \alpha_{j}^{(2)}+2\left(2 \delta^{s}+1\right),}
\end{gathered}
$$

where

$$
\begin{gathered}
\alpha^{s} \sum_{i=1}^{2} \alpha_{i}^{(2) 2}+\beta^{s} \sum_{i=1}^{2} \alpha_{i}^{(2)}-\left(2 \delta^{s}+1\right)=0 \\
\delta^{s} \sum_{i=1}^{2} \alpha_{i}^{(2) 2}-\left(\beta^{s} \sum_{i=1}^{2} \alpha_{i}^{(2)}+1\right) \sum_{j<k}^{2} \alpha_{j}^{(2)} \alpha_{k}^{(2)}-2 \alpha^{s} \sum_{j<k}^{2} \alpha_{j}^{(2) 2} \alpha_{k}^{(2) 2}=0
\end{gathered}
$$

The energy eigenvalue therefore is

$$
\begin{aligned}
& E_{2 \kappa}^{S \pm}=\frac{1}{2}\left\{C_{s} \pm\left[C_{s}^{2}+4 M^{2}-4 M C_{s}-8 \kappa A+4 A+8 A B+24 \sqrt{a_{1}^{s}}\right.\right. \\
& \left.\left.+4 \sqrt{a_{1}^{s}} \sqrt{4 \kappa^{2}+4 \kappa+1-4 d_{1}^{s}}-4 \beta^{s 2}\right]^{1 / 2}\right\} .
\end{aligned}
$$

For the upper component of the wave function, we have

$$
F_{2 \kappa}^{S}(r)=N_{2 \kappa} \prod_{i=1}^{2}\left(r-\alpha_{i}^{(2)}\right) r^{(1 / 2)\left(1+\sqrt{\left.(2 \kappa+1)^{2}-4 d_{1}^{s}\right)}\right.} \exp \left(-\frac{1}{2} \sqrt{a_{1}^{s}} r^{2}-\frac{b_{1}^{s}}{2 \sqrt{a_{1}^{s}}} r\right)
$$

We have given some numerical values of the energy eigenvalues in Tables 1, 2, 3, 4, 5, and 6 for various states. For the final point, we wish to emphasize on the degeneracy-removing role of the (Cornell) tensor potential. As we already know, for vanishing tensor interaction $(A=B=0)$, the pseudospin doublets, that is, states with quantum numbers $(n, l, j=l+1 / 2)$ and $(n-1, l+2, j=l+3 / 2)$ are degenerate. The degenerate states in the spin doublets are those with quantum numbers $(n, \ell, j=\ell+1 / 2)$ and $(n, \ell, j=\ell-1 / 2)$, where $n, l$, and $j$ 
Table 1: Bound state for the pseudospin symmetry $b=1, M=1 \mathrm{fm}^{-1}, C_{\mathrm{ps}}=0 \mathrm{fm}^{-1}$.

\begin{tabular}{ccccc}
\hline$\tilde{\ell}$ & $n, \kappa$ & $(\ell, j)$ & $A=B=0$ & $E_{n \kappa}^{\mathrm{ps}^{-}}\left(\mathrm{fm}^{-1}\right)$ \\
\hline 1 & $0,-1$ & $0 S_{1 / 2}$ & -0.882352941 & $A=0.5, B=0.2$ \\
2 & $0,-2$ & $0 P_{3 / 2}$ & -0.945945946 & -1.984891569 \\
3 & $0 d_{5 / 2}$ & -0.969230769 & -2.471004354 \\
4 & $0 f_{7 / 2}-3$ & -0.980198020 & -2.861584264 \\
1 & $0,-4$ & $1 S_{1 / 2}$ & -0.945945946 & -3.199522676 \\
2 & $1,-1$ & $1 p_{3 / 2}$ & -0.969230769 & -2.302587881 \\
3 & $1,-2$ & $1 d_{5 / 2}$ & -0.980198020 & -2.710867336 \\
4 & $1,-3$ & $1 f_{7 / 2}$ & -0.986206897 & -3.060956677 \\
1 & $1,-4$ & $0 d_{3 / 2}$ & -0.945945946 & -3.373431922 \\
2 & 1,2 & $0 f_{5 / 2}$ & -0.969230769 & -1.396649362 \\
3 & 1,3 & $0 g_{7 / 2}$ & -0.980198020 & -1.411440323 \\
4 & 1,4 & $0 h_{9 / 2}$ & -0.986206897 & -1.414060440 \\
\hline
\end{tabular}

Table 2: Bound state for the spin symmetry $b=1, M=1 \mathrm{fm}^{-1}, C_{s}=0 \mathrm{fm}^{-1}$.

\begin{tabular}{ccccc}
\hline$\ell$ & $n, \kappa$ & $(\ell, j)$ & $A=B=0$ & $E_{n \kappa}^{s^{+}}\left(\mathrm{fm}^{-1}\right)$ \\
\hline 1 & $0,-2$ & $0 p_{3 / 2}$ & 0.882352941 & $A=0.5, B=0.2$ \\
2 & $0,-3$ & $0 d_{5 / 2}$ & 0.945945946 & 2.408312542 \\
3 & $0,-4$ & $0 f_{7 / 2}$ & 0.969230769 & 2.835633655 \\
4 & $0 g_{9 / 2}$ & 0.980198020 & 3.186164523 \\
1 & $1,-5$ & $1 p_{3 / 2}$ & 0.945945946 & 3.494741008 \\
2 & $1,-3$ & $1 d_{5 / 2}$ & 0.969230769 & 2.557131582 \\
3 & $1,-4$ & $1 f_{7 / 2}$ & 0.980198020 & 2.960686798 \\
4 & $1,-5$ & $1 g_{9 / 2}$ & 0.986206897 & 3.301543491 \\
1 & 0,1 & $0 p_{1 / 2}$ & 0.882352941 & 3.603991399 \\
2 & 0,2 & $0 d_{3 / 2}$ & 0.945945946 & 1.577184026 \\
3 & $0 f_{5 / 2}$ & 0.969230769 & 1.665371674 \\
4 & 0,3 & 0.980198020 & 1.695353106 \\
1 & 0,4 & $1 g_{7 / 2}$ & 0.945945946 & 1.708910609 \\
2 & 1,1 & $1 d_{3 / 2}$ & 0.969230769 & 1.798483171 \\
3 & 1,2 & $1 f_{5 / 2}$ & 0.980198020 & 1.873601141 \\
4 & 1,3 & $1 g_{7 / 2}$ & 0.986206897 & 1.910316883 \\
\hline
\end{tabular}

Table 3: Energies in the pseudospin symmetry Limit for $A=0.5, B=0.2, b=1, M=1 \mathrm{fm}^{-1}$.

\begin{tabular}{lcccc}
\hline$C_{\mathrm{ps}}$ & $1 P_{3 / 2}$ & $1 f_{7 / 2}$ & $E_{n \kappa}^{\mathrm{ps}^{-}}\left(\mathrm{fm}^{-1}\right)$ & $0 d_{3 / 2}$ \\
\hline-4 & -4.720070702 & -5.340430189 & -3.401741077 & $0 g_{7 / 2}$ \\
-2 & -3.528809436 & -4.224630585 & -1.997983133 & -3.398579611 \\
0 & -2.710867336 & -3.373431922 & -1.396649362 & -1.989883697 \\
2 & -2.197514516 & -2.788106698 & -1.161286022 & -1.224585994 \\
4 & -1.872532099 & -2.315650253 & -1.021036465 & -1.131350750 \\
\hline
\end{tabular}


Table 4: Energies in the pseudospin symmetry limit for $A=0.5, B=0.2, b=1, C_{\mathrm{ps}}=0 \mathrm{fm}^{-1}$.

\begin{tabular}{lcccc}
\hline$M\left(\mathrm{fm}^{-1}\right)$ & \multicolumn{3}{c}{$E_{n \kappa}^{\mathrm{ps}^{-}}\left(\mathrm{fm}^{-1}\right)$} & $0 d_{3 / 2}$ \\
\hline 0 & $1 P_{3 / 2}$ & $1 f_{7 / 2}$ & -0.997983133 & -0.989883697 \\
2 & -2.528809436 & -3.224630585 & -2.161286022 & -2.224585997 \\
4 & -3.197514516 & -3.788106698 & -3.914496549 & -4.070243046 \\
6 & -4.653565405 & -5.108745157 & -5.75746618 & -5.983999509 \\
\hline
\end{tabular}

Table 5: Energies in the spin symmetry limit for $A=0.5, B=0.2, b=1, M=1 \mathrm{fm}^{-1}$.

\begin{tabular}{lcccc}
\hline$C_{s}$ & & \multicolumn{2}{c}{$E_{n \kappa}^{s^{+}}\left(\mathrm{fm}^{-1}\right)$} & \\
& $0 f_{7 / 2}$ & $1 d_{5 / 2}$ & $0 P_{1 / 2}$ & $1 f_{5 / 2}$ \\
\hline-4 & 2.202940158 & 1.991277770 & 0.885667514 & 1.319214847 \\
-2 & 2.599395981 & 2.379236118 & 1.159816872 & 1.527861114 \\
0 & 3.186164523 & 2.960686798 & 1.577184026 & 1.910316883 \\
2 & 4.044444399 & 3.826180645 & 2.362620095 & 2.672956317 \\
4 & 5.214113098 & 5.019180712 & 3.720450502 & 3.966069464 \\
\hline
\end{tabular}

Table 6: Energies in the spin symmetry limit for $A=0.5, B=0.2, b=1, C_{s}=0 \mathrm{fm}^{-1}$.

\begin{tabular}{lcccc}
\hline$M\left(\mathrm{fm}^{-1}\right)$ & \multicolumn{3}{c}{$E_{n \kappa}^{s^{+}}\left(\mathrm{fm}^{-1}\right)$} & \\
\hline 0 & $0 f_{7 / 2}$ & $1 d_{5 / 2}$ & 1.362620095 & 1.672956317 \\
2 & 3.044444399 & 2.826180645 & 2.159816872 & 2.527861114 \\
4 & 3.599395981 & 3.379236118 & 3.669041728 & 4.186965577 \\
6 & 4.926530873 & 4.721303277 & 5.304646313 & 6.017810527 \\
\hline
\end{tabular}

are the radial, the orbital, and the total angular momentum quantum numbers, respectively (see Tables 1 and 2). Our numerical data reveals that, in the pseudospin symmetry limit, the degenerate states for $(A=B=0)$ are $n s_{1 / 2},(n-1) d_{3 / 2}$ for $\widetilde{\ell}=1(\ell=0), n p_{3 / 2},(n-1) f_{5 / 2}$ for $\tilde{\ell}=2(\ell=1), n d_{5 / 2},(n-1) g_{7 / 2}$ for $\tilde{\ell}=3(\ell=2), n f_{7 / 2},(n-1) h_{9 / 2}$ for $\tilde{\ell}=4(\ell=3)$, and so forth. For spin symmetry limit and $(A=B=0)$ one can clearly see that the degeneracy occurs in $\left(n p_{1 / 2}, n p_{3 / 2}\right)$ for $\ell=1,\left(n d_{3 / 2}, n d_{5 / 2}\right)$ for $\ell=2,\left(n f_{5 / 2}, n f_{7 / 2}\right)$ for $\ell=3,\left(n g_{7 / 2}, n g_{9 / 2}\right)$ for $\ell=4$, and so forth.

\section{Conclusion}

Because of the established roles of the Cornell potential and spin, and pseudospin symmetries in nuclear and hadrons spectroscopy, we solved the Dirac equation under these symmetry limits for vector, scalar, and tensor interactions of Cornell-type. In our calculations, on the one hand, due to the failure of other common analytical techniques, and, on the other hand, the better insight which analytical techniques provide us in comparison with their numerical counterparts, we used the quasianalytical ansatz approach. By proposing novel physical solutions and after lengthy calculations, we could find the arbitrary-state solutions. Our results clearly show the degeneracy-removing role of the tensor term and provide the requisite understanding of the solutions for possible further studies. Both the 
energy spectrum and the eigenfunctions can be used in related systems after the proper phenomenological fits done.

\section{References}

[1] D. H. Perkins, An Introduction to High Energy Physics, Cambridge University Press, 2000.

[2] J. N. Ginocchio, "Relativistic symmetries in nuclei and hadrons," Physics Reports, vol. 414, no. 4-5, pp. 165-261, 2005.

[3] J. N. Ginocchio and A. Leviatan, "On the relativistic foundations of pseudospin symmetry in nuclei," Physics Letters B, vol. 425, no. 1-2, pp. 1-5, 1998.

[4] G. Mao, "Effect of tensor couplings in a relativistic Hartree approach for finite nuclei," Physical Review C, vol. 67, no. 4, Article ID 044318, 12 pages, 2003.

[5] P. Alberto, R. Lisboa, M. Malheiro, and A. S. de Castro, “Tensor coupling and pseudospin symmetry in nuclei," Physical Review C, vol. 71, no. 3, Article ID 034313, 7 pages, 2005.

[6] R. J. Furnstahl, J. J. Rusnak, and B. D. Serot, "The nuclear spin-orbit force in chiral effective field theories," Nuclear Physics A, vol. 632, no. 4, pp. 607-623, 1998.

[7] G.-F. Wei and S.-H. Dong, "Approximately analytical solutions of the Manning-Rosen potential with the spin-orbit coupling term and spin symmetry," Physics Letters A, vol. 373, no. 1, pp. 49-53, 2008.

[8] G. F. Wei and S. H. Dong, "Algebraic approach to pseudospin symmetry for the Dirac equation with scalar and vector modified Pöschl-Teller potentials," Europhysics Letters, vol. 87, no. 4, article 40004, 2009.

[9] G. F. Wei and S. H. Dong, "Spin symmetry in the relativistic symmetrical well potential including a proper approximation to the spin-orbit coupling term," Physica Scripta, vol. 81, no. 3, Article ID 035009, 2010.

[10] G. F. Wei and S. H. Dong, "Pseudospin symmetry for modified Rosen-Morse potential including a Pekeris-type approximation to the pseudo-centrifugal term," European Journal of Physics A, vol. 46, no. 2, pp. 207-212, 2010.

[11] G. F. Wei and S. H. Dong, "Pseudospin symmetry in the relativistic Manning-Rosen potential including a Pekeris-type approximation to the pseudo-centrifugal term," Physics Letters B, vol. 686, no. 4-5, pp. 288-292, 2010.

[12] W.-C. Qiang and S.-H. Dong, "SUSYQM and SWKB approaches to the relativistic equations with hyperbolic potential $V_{0}$ tanh $^{2}$ (r/d)," Physica Scripta, vol. 72, no. 2-3, pp. 127-131, 2005.

[13] S. Zarrinkamar, A. A. Rajabi, and H. Hassanabadi, "Dirac equation for the harmonic scalar and vector potentials and linear plus Coulomb-like tensor potential; the SUSY approach," Annals of Physics, vol. 325, no. 11, pp. 2522-2528, 2010.

[14] O. Aydoğdu and R. Sever, "Pseudospin and spin symmetry in Dirac-Morse problem with a tensor potential," Physics Letters. B, vol. 703, no. 3, pp. 379-385, 2011.

[15] Y. Xu, S. He, and C.-S. Jia, "Approximate analytical solutions of the Dirac equation with the PöschlTeller potential including the spin-orbit coupling term," Journal of Physics A, vol. 41, no. 25, article 255302, 2008.

[16] M. R. Setare and Z. Nazari, "Pseudospin symmetry in deformed nuclei with axially-symmetric harmonic oscillator potential," Modern Physics Letters A, vol. 25, no. 549, 2010.

[17] K. J. Oyewumi and C. O. Akoshile, "Bound-state solutions of the Dirac-Rosen-Morse potential with spin and pseudospin symmetry," European Physical Journal A, vol. 45, no. 3, pp. 311-318, 2010.

[18] E. Maghsoodi, H. Hassanabadi, and O. Aydogdu, "Dirac particles in the presence of the Yukawa potential plus a tensor interaction in SUSYQM framework," Physica Scripta, vol. 86, Article ID 015005, 2012.

[19] H. Hassanabadi and A. A. Rajabi, "Energy levels of a spherical quantum dot in a confining potential," Physics Letters A, vol. 373, no. 6, pp. 679-681, 2009.

[20] H. Hassanabadi and A. A. Rajabi, "Relativistic versus nonrelativistic solution of the N-fermion problem in a hyperradius-confining potential," Few-Body Systems, vol. 41, no. 3-4, pp. 201-210, 2007.

[21] S.-H. Dong, Z.-Q. Ma, and G. Esposito, "Exact solutions of the Schrödinger equation with inversepower potential," Foundations of Physics Letters, vol. 12, no. 5, pp. 465-474, 1999.

[22] S.-H. Dong, "Exact solutions of the two-dimensional Schrödinger equation with certain central potentials," International Journal of Theoretical Physics, vol. 39, no. 4, pp. 1119-1128, 2000.

[23] S. H. Dong, "A new approach to the relativistic Schrödinger equation with central potential: Ansatz method," International Journal of Theoretical Physics, vol. 40, no. 2, pp. 559-567, 2001. 
[24] H. Hassanabadi, H. Rahimov, and S. Zarrinkamar, "Cornell and Coulomb interactions for the Ddimensional Klein-Gordon equation," Annalen der Physik, vol. 523, no. 7, pp. 566-575, 2011.

[25] D. Agboola and Y. Zhang, "Unified derivation of exact solutions for a class of quasi-exactly solvable models," Journal of Mathematical Physics, vol. 53, Article ID 042101, 13 pages, 2012.

[26] H. Hassanabadi, B. H. Yazarloo, S. Zarrinkamar, and A. A. Rajabi, “Duffin-Kemmer-Petiau equation under a scalar Coulomb interaction," Physical Review C, vol. 84, Article ID 064003, 4 pages, 2011.

[27] S. M. Ikhdair, "Bound state energies and wave functions of spherical quantum dots in presence of aconfining potential model," http:/ / arxiv.org/abs/1110.0340.

[28] M. Znojil, "Singular anharmonicities and the analytic continued fractions," Journal of Mathematical Physics, vol. 30, no. 1, pp. 23-27, 1989.

[29] M. Znojil, "The generalized continued fractions and potentials of the Lennard-Jones type," Journal of Mathematical Physics, vol. 31, no. 1955, 7 pages, 1990. 

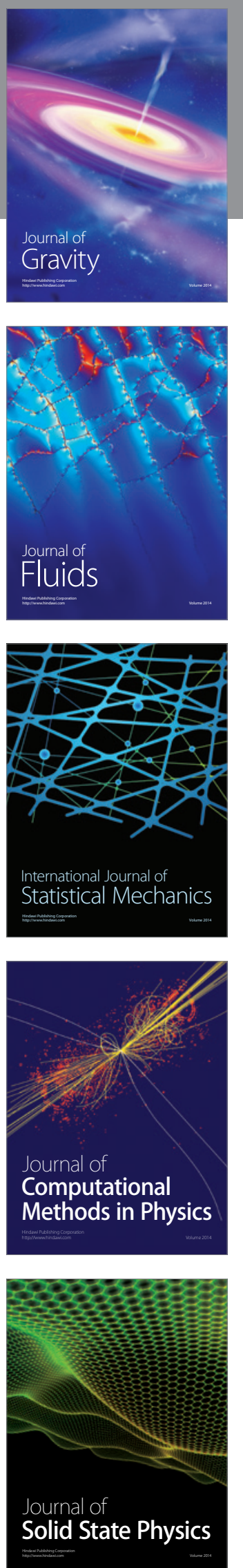

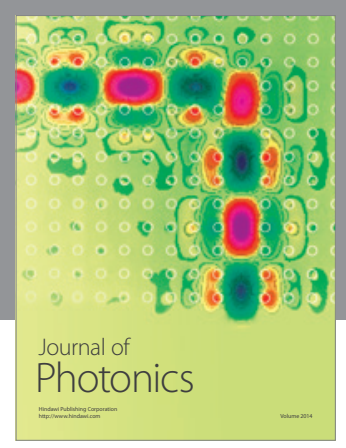

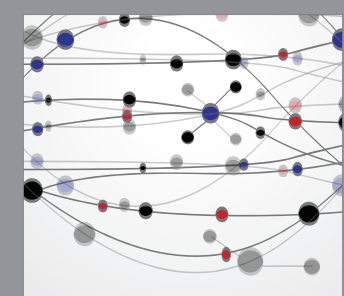

The Scientific World Journal
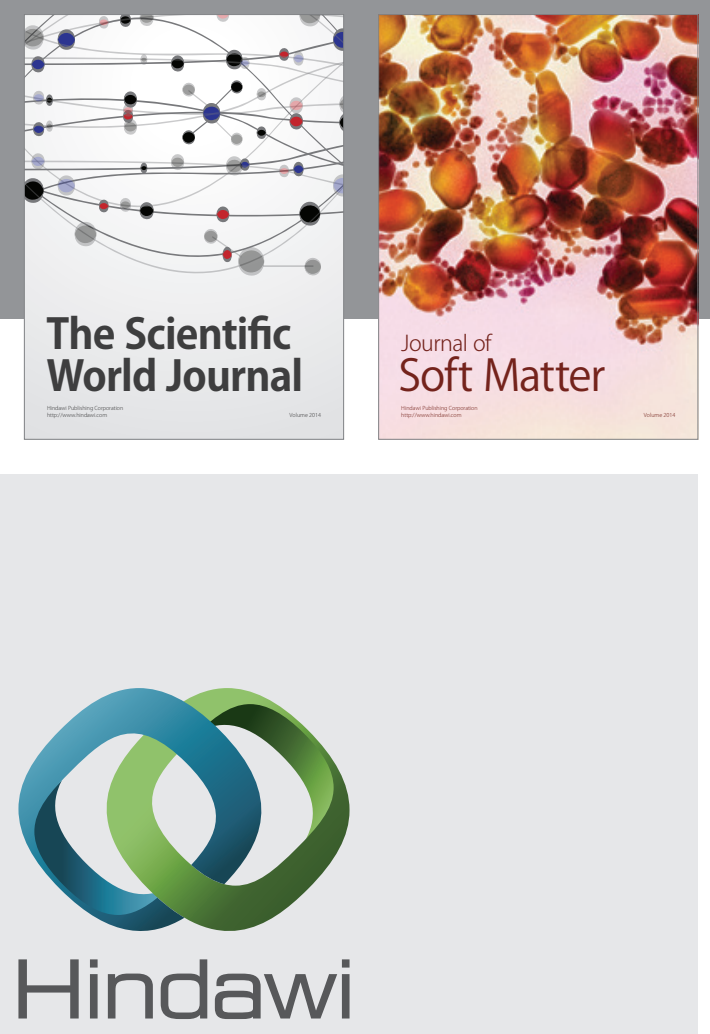

Submit your manuscripts at

http://www.hindawi.com
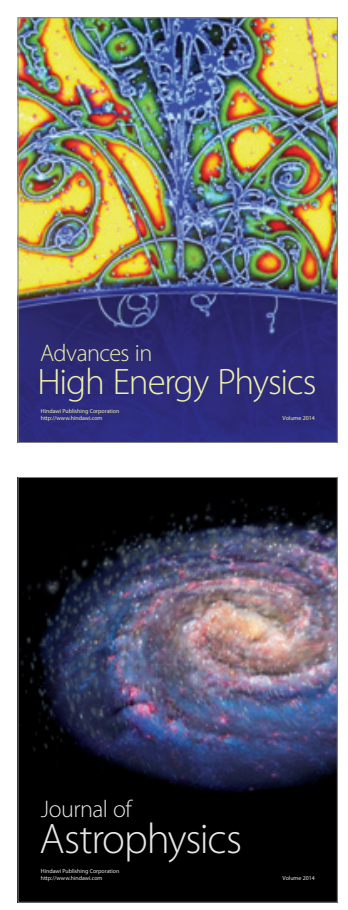
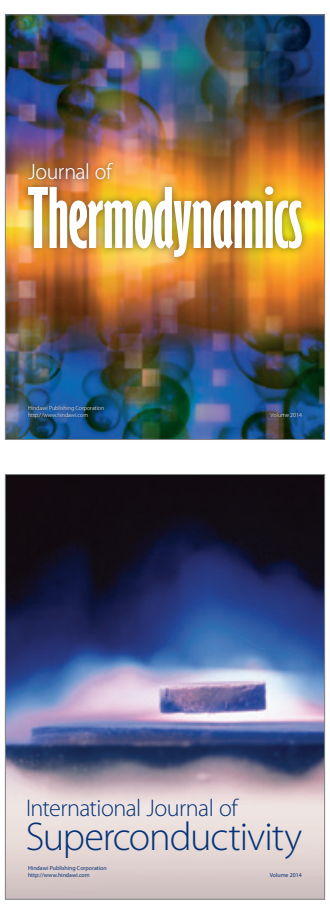
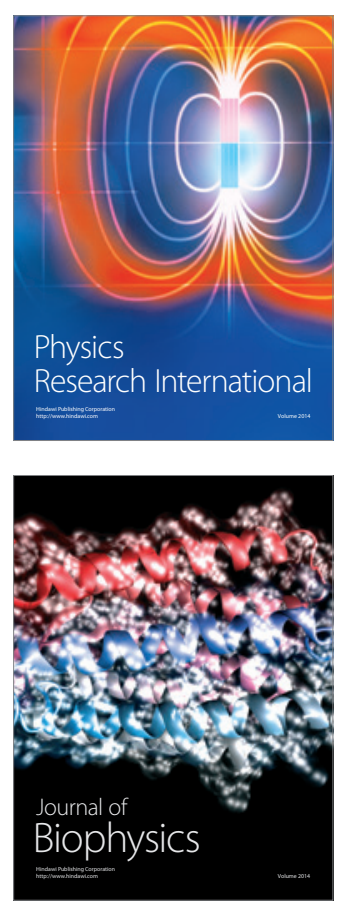
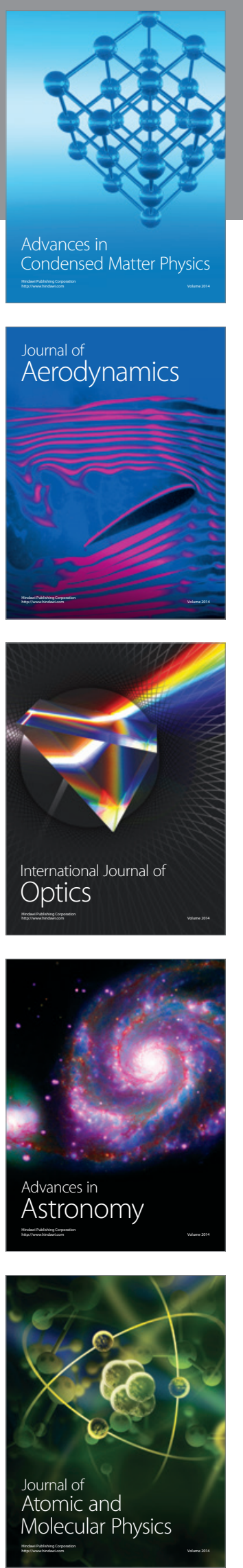